\title{
Pour une approche contrastive du présent simple en français et en portugais
}

\author{
Sílvia Araújo \\ Université du Minho, Portugal \\ saraujo@ilch.uminho.pt \\ Otília Sousa \\ École Supérieure d'Éducation de Lisbonne, Portugal \\ otilias@eselx.ipl.pt
}

\section{Introduction}

Les temps du passé ont toujours constitué une pierre d'achoppement aussi bien dans l'enseignement du français aux étudiants lusophones que dans celui du portugais aux francophones. Le flot continu d'articles sur ce sujet attesterait, à lui seul, la difficulté du problème. Mais aussi étonnant que cela puisse paraître, il n'existe à ce jour aucune étude systématique qui aborde sur une base théorique le présent simple dans le passage du français vers le portugais et/ou vice-versa. Or, une telle étude nous paraît une voie de recherche intéressante et fructueuse qui ouvre de nombreuses perspectives de recherche, dont les possibilités d'application, que ce soit au domaine de l'enseignement des langues étrangères ou celui de la traduction, ne sont pas négligeables. Cette réflexion est d'autant plus urgente que l'emploi du présent simple pose de sérieux problèmes aux élèves lusophones plus inexpérimentés. En effet, ces derniers ont parfois beaucoup de peine à trouver les bonnes correspondances du présent simple en portugais.

Afin d'aboutir à des conclusions fiables sur les similarités et les divergences entre le français et le portugais quant aux conditions d'apparition et au mode de fonctionnement du présent simple, nous nous attacherons, dans l'étude contrastive qui suit, à examiner des échantillons de textes littéraires français traduits en portugais et des extraits de textes littéraires portugais traduits en français ${ }^{1}$. Il s'agira essentiellement de montrer, en recourant à la Théorie des Opérations Énonciatives d'Antoine Culioli (Culioli, 1990; Campos \& Xavier, 1991; Campos, 1997, 1998, entre autres), par quels moyens linguistiques le portugais exprime les valeurs aspectuo-temporelles associées au présent simple en français.

\section{Quelques précisions théoriques}

\section{1 À propos de la catégorie grammaticale du temps}

Dans notre analyse qui s'inscrit dans le cadre d'une théorie énonciative, nous considérons que chaque procès est représentable sur une droite continue où se projette la classe des instants construite par chaque énonciation, chronologiquement orientée de gauche à droite. Cette représentation est faite en termes d'intervalles (succession continue d'instants), qui peuvent être ouverts, semi-ouverts ou fermés, et qui sont repérés dans des systèmes de coordonnées énonciatives créés par l'énonciation même (Campos, 1997: 54). Ainsi, les paramètres sujet énonciateur $S_{0}$ et temps ou 'moment' $T_{0}$ de l'énonciation définissent un objet métalinguistique Sit $\left(\mathrm{S}_{0}, \mathrm{~T}_{0}\right)$ ou plus simplement $\mathrm{Sit}_{0}$, situation d'énonciation origine, par rapport à laquelle sont repérées, entre autres, les coordonnées de l'événement Sit $\left(\mathrm{S}_{2}, \mathrm{~T}_{2}\right)$ ou $\mathrm{Sit}_{2}$. La valeur aspectuo-temporelle (voir Benveniste, 1974: 74 et ss.; Campos \& Xavier, 1991: 300-303) d'un énoncé résulte donc bel et bien de la mise en relation (ou 'repérage') du moment auquel réfère l'énoncé (ou 
temps de validation de la relation prédicative $\left(\mathrm{T}_{2}\right)$ ) avec le moment de l'énonciation (ou "repère-origine du temps" noté $\mathrm{T}_{0}$ ). Cette opération de repérage peut avoir trois valeurs:

- si l'événement que décrit le prédicat est en cours en $\mathrm{T}_{0}$, on a un repérage à valeur d'identification $\left(\mathrm{T}_{2}\right.$ $\left.=\mathrm{T}_{0}\right)$;

- si l'événement décrit par le prédicat se passe antérieurement ou postérieurement à $\mathrm{T}_{0}$, on aura alors un repérage à valeur de différenciation $\left(\mathrm{T}_{2} \neq \mathrm{T}_{0}\right)$;

- Si l'événement que décrit le prédicat n'a aucun lien avec $\mathrm{T}_{0}$, on a alors un repérage à valeur de rupture $\left(\mathrm{T}_{2} \omega \mathrm{T}_{0}\right)$.

\section{2 À propos de la catégorie grammaticale de l'aspect}

Emprunté de la description du grec et des langues slaves et sémitiques, on doit reconnaître que le terme d'aspect a effectivement été utilisé en français, tout comme en portugais, pour nommer un peu n'importe quoi. Rappelons, tout de même, que les valeurs sémantiques attachées aux jeux d'oppositions (comme achevé/inachevé, accompli/inaccompli, résultatif/non-résultatif, processus/état, etc.) que l'on retrouve dans la littérature sur l'aspect (cf. par ex. Comrie, 1976) résultent, en fait, d'opérations qui ne sont pas propres à cette catégorie: c'est la façon dont, à travers des règles explicitables, la catégorie 'aspect' spécifie et se trouve en retour spécifiée par d'autres catégories (telles que la diathèse ou la modalité) qui permet d'appréhender la signification d'un énoncé dans un contexte donné. Nous retrouvons ici l'idée que l'aspect est une catégorie pluridimensionnelle et que, par conséquent, il ne s'agit pas de recréer une taxinomie statique, dans laquelle les procès seraient classés une fois pour toutes, comme une donnée de départ connue et stable.

La typologie que nous adopterons ici pose, à la suite de Culioli (1991-92: 7-15), que les types de procès issus de la détermination verbale correspondent aux trois catégories posées pour rendre compte de phénomènes équivalents dans la détermination nominale: discret, dense, compact ${ }^{2}$ (cf. entre autres, Franckel, Paillard \& de Vogüé, 1988; de Vogüé, 1989; Franckel \& Paillard, 1991; Campos, 1997). L'intérêt de cette typologie nous paraît résider dans le fait qu'elle essaie de se donner des grilles de fonctionnement indépendantes des contraintes syntaxiques qu'elle engendre, ce qui n'est point le cas dans la classification élaborée par Vendler $(1967)^{3}$. Il est intéressant de voir la valeur que prend, par exemple, le présent simple selon qu'on a un type de procès ou un autre dans des constructions syntaxiques apparemment identiques. En effet, on constate que dans l'exemple qui suit:

(1a) $\mathrm{Il} \mathrm{part}^{4}$

le présent donne au verbe une valeur prospective alors que dans un exemple du type:

(2a) Il mange

il renvoie à de l'actuel. En effet, le présent est, dans ce cas, incontestablement plus proche de:

(2b) Il est en train de manger

que:

(3a) Il boit

ne l'est de:

(3b) Il est en train de boire

En effet, il boit s'interprète préférentiellement, sauf contexte contraire, comme 'il est (un) buveur/c'est un ivrogne', glissement impossible avec il mange ${ }^{5}$.

Si l'on tient vraiment à éclairer les variations d'emploi du présent, à la fois dans chacune des deux langues et dans le passage de l'une à l'autre, il nous faut donc tenir compte de ce qui découle des propriétés primitives des procès, sans oublier pour autant que la construction des valeurs temporelles 
soulève des problèmes qui sont de trois ordres: problèmes d'Aktionsart, problèmes de temps et problèmes d'aspect.

\section{3 À propos du présent simple}

La position la plus répandue dans les grammaires du français (Wagner \& Pinchon, 1962: 336; Arrivé et al., 1986: 562) et du portugais (Mateus et al., 1983: 104; Cunha \& Cintra, 1989: 447) consiste à définir le présent simple comme la forme au moyen de laquelle le locuteur exprime tout ce qui constitue son actualité.

Après avoir associé le présent à cette notion d' «actuel», ces grammaires dressent une liste pour indiquer les multiples valeurs que peut prendre, en contexte, ce temps verbal. Voici, sous la forme d'un tableau comparatif simplifié, un résumé de ce qu'on peut trouver dans ces grammaires:

\begin{tabular}{|c|c|c|}
\hline \multirow[t]{2}{*}{ Valeurs } & \multicolumn{2}{|l|}{ Exemples } \\
\hline & Français $^{6}$ & Portugais \\
\hline $\begin{array}{l}\text { actuelle/«procès en } \\
\text { cours» }\end{array}$ & $\begin{array}{l}\text { (4) Ne le dérange pas, il téléphone (il } \\
\text { est au téléphone) } \\
\text { (5a) Il travaille actuellement } \\
\text { (6) Chut! Pierre dort }\end{array}$ & $\begin{array}{l}\text { (14) O céu está limpo, não há } \\
\text { nenhuma nuvem acima de nós } \\
\text { (15) Trago uma saia }\end{array}$ \\
\hline $\begin{array}{l}\text { générique/itérative } \\
\text { /habituelle }\end{array}$ & $\begin{array}{l}\text { (7) L'eau bout à } 100 \text { degrés } \\
\text { (8) Henri boit } \\
\text { (9) Il se couche tôt } \\
\text { (10) Il vient souvent à la maison } \\
\text { (11a) Je pars tous les matins à } 7 \text { heures }\end{array}$ & $\begin{array}{l}\text { (16) A água ferve a } 100 \text { graus } \\
\text { (17) O Pedro fuma/bebe } \\
\text { (18) Como pouquíssimo } \\
\text { (19) Eu na sua igreja não mando: só } \\
\text { assisto e apoio } \\
\text { (20) Ele compra o jornal todas as } \\
\text { semanas }\end{array}$ \\
\hline prospective & $\begin{array}{l}\text { (12) J'arrive } \\
\text { (11b) Je pars demain matin }\end{array}$ & $\begin{array}{l}\text { (21) Vou para Coimbra } \\
\text { (22) Desço na próxima estação }\end{array}$ \\
\hline $\begin{array}{l}\text { narrative ou } \\
\text { historique }\end{array}$ & $\begin{array}{l}\text { (13) Pas plus tard qu'hier, je pars de } \\
\text { chez moi sans prendre les clés, je fais } \\
10 \text { kilomètres et je tombe en panne } \\
\text { d'essence }^{8}\end{array}$ & $\begin{array}{l}\text { (23) A avenida é o mar dos foliões. } \\
\text { Serpentinas cortam o ar carregado de } \\
\text { éter, rolam das sacadas, pendem das } \\
\text { árvores e dos fios, unem com os seus } \\
\text { matizes os automóveis do corso [...]. }\end{array}$ \\
\hline
\end{tabular}

Tableau 1. Valeurs de base du présent simple en français et en portugais dans les grammaires

Un survol rapide de ce tableau montre que les descriptions sommaires du présent simple proposées dans les grammaires de l'une et l'autre langue ne permettent guère de les différencier. En effet, ce temps verbal semble donner lieu, dans les deux langues, à des emplois relativement similaires. Cependant, si l'on regarde de plus près, on s'aperçoit que les exemples à valeur de procès en cours soulèvent des problèmes supplémentaires qui doivent être obligatoirement résolus lorsqu'on désire enseigner à des étrangers le maniement des temps verbaux. En effet, si l'on compare les énoncés français ((4)-(6)), où l'on a affaire à des procès de type processus (à fonctionnement dense ${ }^{9}$, comme nous verrons plus loin), on constate que le portugais est ici quasiment contraint d'avoir recours à des verbes tels que estar et haver (cf. supra, ex. (14)), qui renvoient à des procès de type statif repérés par rapport à $\mathrm{T}_{0}$. Quant à l'ex. (15), il nous faut préciser que le verbe trago est, lui aussi, statif en ce sens où l'événement trago uma saia (je porte une robe, en français) focalise l'attention sur l'état résultant, qui est un état-présent concomitant à $\mathrm{T}_{0}$, et non sur l'événement qui lui est immédiatement antérieur «vestir uma saia» (mettre une robe).

Dans les deux langues, le présent simple présente visiblement une très large variété d'emplois qui n'apparaît pas facilement réductible, a priori, à une caractérisation générale et invariante. Notre étude a justement pour objectif de dégager une unité dans la diversité des emplois et des valeurs du présent simple et d'expliquer comment se relient et s'enchaînent ces valeurs. Pour cela, nous retiendrons comme 
caractérisation de la forme de présent simple la valeur d'indétermination (Paillard, 1988; Serbat, 1988, Franckel, 1989), qui est celle qui nous paraît le mieux à même de rendre compte des emplois du présent en français et en portugais. Une fois qu'on reconnaît que le présent simple ne construit pas la distinction d'un t particulier et ne permet donc pas de localiser le procès p (Paillard, 1988: 100-101; Franckel, 1989: 83) sur la classe des instants, on ne s'étonne plus de le voir tour à tour apparaître dans les contextes les plus divers, quel que soit le point de référence - présent, passé ou fictif.

\section{Zones de convergence et de divergence en ce qui concerne les valeurs de base du présent simple en français et en portugais}

Pour rendre compte de cette gamme de valeurs inhérentes au présent simple dans les deux langues cidessus, nous nous proposons de partir de l'analyse d'un échantillon d'exemples dont la représentativité devra être justifiée a posteriori par la possibilité contrôlée de rapporter tel ou tel énoncé à tel ou tel type d'opération. Il est à noter, d'ores et déjà, que ce sont les valeurs qui dérivent d'un repérage par identification stricte ou d'un repérage par rupture par rapport à $\mathrm{T}_{0}$ qui posent le moins de problèmes lorsqu'il s'agit de passer du français au portugais ou vice-versa, comme en témoigne le tableau suivant où l'on présente quelques exemples en français avec leur traduction littérale en portugais:

\begin{tabular}{|c|c|c|c|c|}
\hline Opérations & Type de procès & Valeurs & Français & Portugais \\
\hline \multirow{3}{*}{$\begin{array}{l}\text { identification } \\
\text { entre } T_{2} \text { et } T_{0}\end{array}$} & $\begin{array}{l}\text { verbes de } \\
\text { perception }\end{array}$ & \multirow{3}{*}{$\begin{array}{l}\text { procès en } \\
\text { cours }\end{array}$} & $\begin{array}{l}\text { (24a) J'entends un } \\
\text { bruit } \\
\text { (25a) Sinto uma dor }\end{array}$ & $\begin{array}{l}\text { (24b) Ouço um barulho } \\
\text { (25b) Je sens une } \\
\text { douleur }\end{array}$ \\
\hline & verbes performatifs & & $\begin{array}{l}\text { (26a) Je déclare } \\
\text { ouverte la session }\end{array}$ & $\begin{array}{l}\text { (26b) Declaro aberta a } \\
\text { sessão }\end{array}$ \\
\hline & verbes d'ostension & & (27a) Regarde! & (27b) Olha! \\
\hline \multirow{3}{*}{$\begin{array}{l}\text { rupture entre } \\
T_{2} \text { et } T_{0}\end{array}$} & \multirow{3}{*}{$\begin{array}{l}\text { tous les types de } \\
\text { procès }\end{array}$} & générique & $\begin{array}{l}\text { (28a) L'eau bout à } 100 \\
\text { degrés }\end{array}$ & $\begin{array}{l}\text { (28b) A água ferve a } \\
100 \text { graus }\end{array}$ \\
\hline & & habituelle & (29a) Jean fume & (29b) O João fuma \\
\hline & & itérative & $\begin{array}{l}\begin{array}{l}(30 \mathrm{a}) \text { Il achète } \\
\text { journal } \\
\text { semaines }\end{array} \\
\text { toutes }\end{array}$ & $\begin{array}{l}\text { (30b) Ele compra o } \\
\text { jornal todas as semanas }\end{array}$ \\
\hline
\end{tabular}

Tableau 2: Zones de convergence du présent simple dans les deux langues

Ce tableau fait apparaître une similarité globale de fonctionnement entre les deux langues pour ce qui est des contextes qui admettent la lecture générique/habituelle (i.e. tous les contextes qui dénotent un procès valable pour une série illimitée de moments) et pour ceux qui impliquent la synchronicité entre les deux paramètres $\left(\mathrm{T}_{2}\right.$ et $\left.\mathrm{T}_{0}\right)$ en ce sens où il $\mathrm{y}$ a rattachement explicite du procès à un point précis du temps, à savoir le moment de l'énonciation. Avec les verbes performatifs, c'est le présent de l'énonciation stricto sensu qui entre en jeu. En effet, on a affaire à une identification d'autant plus stricte que l'actualisation totale de l'événement décrit et le fait de le dire occupent clairement le même espace temporel $\left(\mathrm{T}_{0}\right)$. Dans tous ces cas de figure, on peut constater que le passage d'une langue à l'autre peut se faire en recourant à la traduction littérale. Par contre, celle-ci semble bien plus contrainte quand on affaire à des énoncés dont la valeur est purement processive, quel que soit le type de procès concerné et pour ce qui est du mode de repérage par différenciation par rapport à $\mathrm{T}_{0}$ : 


\begin{tabular}{|c|c|c|c|c|}
\hline Opérations & $\begin{array}{l}\text { Type de } \\
\text { procès }\end{array}$ & Valeurs & Français & Portugais \\
\hline \multirow{3}{*}{$\begin{array}{l}\text { identification } \\
\left(\mathrm{T}_{2}=\mathrm{T}_{0}\right)\end{array}$} & $\begin{array}{l}\text { procès de } \\
\text { type dense }\end{array}$ & \multirow{3}{*}{$\begin{array}{l}\text { procès en } \\
\text { cours }\end{array}$} & (31a) Il dort & $\begin{array}{l}(31 \mathrm{~b}) \text { ele *dorme/está a } \\
\text { dormir }\end{array}$ \\
\hline & $\begin{array}{l}\text { procès de } \\
\text { type dense } \\
\text { discrétisé }\end{array}$ & & $\begin{array}{l}(32 \mathrm{a}) \text { Marie lit un } \\
\text { roman }\end{array}$ & $\begin{array}{l}\text { (32b) A Maria *lê/está } \\
\text { a ler um romance }\end{array}$ \\
\hline & $\begin{array}{l}\text { procès de } \\
\text { type discret } \\
\text { (à bornes } \\
\text { confondues) }\end{array}$ & & $\begin{array}{l}\text { (33a) Il arrive (je } \\
\text { l'aperçois déjà) }\end{array}$ & $\begin{array}{l}\text { (33b) Ele *chega/está a } \\
\text { chegar (Já o vejo) }\end{array}$ \\
\hline \multirow[t]{2}{*}{$\begin{array}{l}\text { différenciation } \\
\left(T_{2} \neq T_{0}\right)\end{array}$} & \multirow[t]{2}{*}{$\begin{array}{l}\text { procès de } \\
\text { type discret } \\
\text { (à bornes } \\
\text { confondues) }\end{array}$} & prospective & $\begin{array}{l}\text { (34a) Ah! Je vais } \\
\text { tomber! } \\
\text { (35a) Ah! Elle va se } \\
\text { noyer! } \\
\text { (36a) (Veuillez } \\
\text { patienter) il arrive }\end{array}$ & $\begin{array}{l}\text { (34b) Ai que caio! } \\
\text { (35b) Ai que se afoga! } \\
\text { (36b) (Espere mais um } \\
\text { pouco) ele *chega/está } \\
\text { a chegar }\end{array}$ \\
\hline & & rétrospective & $\begin{array}{l}\text { (37a) Je sors de chez le } \\
\text { dentiste } \\
(38 a) \text { Je rentre à } \\
\text { l'instant de la faculté }\end{array}$ & $\begin{array}{l}(37 \mathrm{~b}) * \text { saio/acabo de } \\
\text { sair do dentista } \\
(38 \mathrm{~b}) * \text { chego/acabo de } \\
\text { chegar da faculdade }\end{array}$ \\
\hline
\end{tabular}

Tableau 3. Zones de divergence du présent simple dans les deux langues

Notons, tout d'abord, que le présent simple n'est pas maintenu dans la traduction du français vers le portugais, lorsqu'il dénote un procès unique pris dans son déroulement ou bien lorsqu'il peut être considéré comme la trace d'une opération de différenciation en ce sens où il confère aux énoncés une valeur prospective ou rétrospective. Il est à noter, tout de même, que le maintien du présent, en portugais, est possible dans quelques énoncés à valeur prospective du type de (34b)-(35b). En (34a), le présent simple est possible en français avec la même valeur prospective ((34a) Ah, Je tombe!). Cela est moins clair pour (35a). Il faut, tout de même, préciser que cette valeur prospective n'est possible, en (34b)-(35b), que parce qu'il s'agit de verbes discrets à bornes confondues pris dans un énoncé au contour prosodique exclamatif. En effet, c'est l'emploi de l'interjection ai! qui sert à repérer spécifiquement le procès par rapport à $\mathrm{T}_{0}$ et c'est donc ce marqueur qui permet l'apparition du présent simple. En supprimant l'interjection mise dans l'entourage contextuel des exemples (34b)-(35b), l'emploi de la périphrase ir + infinitif devient aussitôt obligatoire ((34b') vou cair, (35b') vai afogar-se).

Bien évidemment, il suffit de comparer les exemples français (31a)-(32a) avec les exemples correspondants du portugais $(31 \mathrm{~b})-(32 \mathrm{~b})$ pour conclure que le français connaît cette particularité de pouvoir utiliser le présent simple là où le portugais ne peut utiliser que la forme périphrastique pour construire la valeur de procès en cours. Il semblerait donc que le présent simple ne soit pas perçu, en portugais, comme marquant de façon suffisamment nette le repérage par identification par rapport à $\mathrm{T}_{0}$, et que, par conséquent, le locuteur natif du portugais préfère une des solutions qui suivent: estar $a+$ infinitif, estar + 'gerúndio, ${ }^{10}$, ir a + infinitif, ir + 'gerúndio' pour véhiculer la valeur progressive. Comme nous le verrons, cette divergence fondamentale entre les deux langues est à l'origine de bon nombre de problèmes de choix de traduction. La fréquence de la première périphrase que nous venons de signaler est la plus élevée. Elle peut être associée à tous les types de procès:

- à des procès de type compact ${ }^{11}$ :

(39) Ela está a gostar da viagem (tr. lit. elle + présent estar ${ }^{12}+$ préposition $a+$ aimer + préposition de + le voyage $\rightarrow$ 'elle est en train d'aimer le voyage') 
(40) Finalmente ela está a saber o que custa a vida! (tr. lit. finalement elle + présent estar + préposition $a+$ savoir + ce que coûte la vie $\rightarrow$ 'Finalement elle est en train de savoir ce que coûte la vie!)

(41) Estou a compreender o que quer dizer (tr. lit. présent estar ( $1^{\text {ère }}$ pers. sing.) + préposition $a$ + comprendre + ce que vous voulez dire $\rightarrow$ 'je suis en train de comprendre ce que vous voulez dire')

- à des procès de type dense:

(42) Ela está a jogar à bola (tr. lit. elle + présent estar + préposition $a+$ jouer + au ballon $\rightarrow$ 'elle est en train de jouer au ballon')

- à des procès de type discret:

(43) Espere um pouco mais, o patrão está a chegar (tr. lit. attendez un peu plus + le patron + présent estar + préposition $a+$ arriver $\rightarrow$ 'attendez un peu plus, le patron est en train d'arriver')

(44) O autocarro está agora a sair da paragem (tr. lit. le bus + présent estar + maintenant + préposition $a+$ sortir + préposition de + l'arrêt $\rightarrow$ 'le bus est maintenant en train de sortir de l'arrêt')

Dans ces deux derniers exemples, la périphrase progressive dilate les procès ponctuels discrets chegar/partir (que Moens \& Steedman (1988) appellent «procès transitoires») de manière à opérer une espèce de déplacement vers l'état préparatoire (non ponctuel) du procès en question. Dès lors, l'exemple (43) admet deux lectures (cf. Campos, 1997: 64): está a chegar (já o vejo) (en fr.: il arrive, je l'aperçois) (valeur de procès en cours) ou está a chegar (não deve faltar muito para que chegue) (en fr.: il arrive, il ne doit pas tarder à arriver) (valeur d'imminence, cf Barroso, 1994: 93). Il en va de même pour l'ex. (44).

Il nous faut souligner, à présent, que seuls les énoncés portugais à valeur prospective peuvent donner lieu à des traductions par la structure analogue en français. Comparons, à titre d'illustration, les paires d'exemples qui suivent:

(45) Vou já / j'arrive tout de suite

(46) Como mais tarde / je mange plus tard

(47) Amanhã trabalho / je travaille demain

(48) Casa-se no próximo ano / il se marie l'année prochaine

(49) Daqui a quatro anos vou ao Brasil / je vais au Brésil dans quatre ans

Tout porte donc à croire que, dans ce cas, les deux langues fonctionnent de façon comparable. Le présent semble pouvoir empiéter sur un futur proche comme simple prolongement de la situation présente (cf. supra, ex. (45)), et ce présent à valeur futurale ne met pas nécessairement en jeu une visée sur l'avenir immédiat du locuteur. Les exemples (48)-(49) en témoignent. Bien évidemment, il nous faut préciser que ce n'est pas le présent, à lui seul, qui situe le procès comme postérieur par rapport à $\mathrm{T}_{0}$. Pour y parvenir, il doit toujours être associé à un marqueur temporel qui projette le procès dans un futur plus ou moins éloigné de $\mathrm{T}_{0}$. En effet, la possibilité que le présent simple du portugais prenne ici une valeur de futur fait apparaître de façon exemplaire le rôle crucial des adverbiaux temporels. Il est intéressant de voir que cette valeur prospective peut, par exemple, être construite à travers le seul marqueur já13 ('déjà') (cf. supra, ex. (45)).

S'il est vrai que le présent peut, en français comme en portugais, dénoter un futur lorsqu'il est combiné à un élément identificateur de futurité, il est vrai aussi que l'emploi du présent simple à valeur rétrospective est nettement plus contraint en portugais. Pour construire cette valeur de «passé récent», le portugais fait appel (cf. supra, exs (37b)-(38b)) à une périphrase aspectuelle, à savoir: acabar de + infinitif ${ }^{14}$ (cf. Laca, 2005: 57), là ou le français emploie le présent simple ${ }^{15}$ (cf. supra, exs (37a)-(38a)): 
(37b) acabo de sair do dentista (tr. lit. je + présent acabar (finir) + préposition de + sortir + préposition de + le dentiste)

(38b) acabo de chegar da faculdade (tr. lit. je + présent acabar (finir) + préposition de + arriver + préposition de + la faculté)

Nous allons maintenant nous intéresser au fonctionnement aoristique du présent simple en français et en portugais. Pour rendre compte du comportement de cette forme en contexte narratif passé, nous allons rendre compte des divergences et convergences observées dans les traductions de romans relevant de chacune de ces deux langues.

\section{Le fonctionnement aoristique du présent français dans le passage d'une langue à l'autre}

Précisons, tout d'abord, que l'observation de notre corpus de textes littéraires fait immédiatement apparaître une similarité globale de fonctionnement entre les deux langues pour ce qui est du mode de décrochage de la fiction. L'énoncé (50a) qui suit avec sa traduction française illustre fort bien le mode de décrochage du récit au présent en français et en portugais:

(50a) A manhã está esta lindeza de Julho, [...], e, se a experiência não engana, vamos ter hoje um dia de calor. Terminada a oração, o almuadem prepara-se para descer, quando de súbito se levanta de baixo um alarido tão [...] espantoso que o cego, assustado, crê em um momento que se desmorona a torre, em outro que estão os malditos cristãos dando assalto às muralhas [...] (História do Cerco de Lisboa (ci-après HCL) de José Saramago, p. 1127)

(50b) C'est un beau matin de juillet, [...], et si l'expérience ne trompe pas, la journée sera chaude aujourd'hui. La prière terminée, le muezzin s'apprête à descendre quand soudain d'en bas s'élève un cri si [...] si épouvantable que l'aveugle, effrayé, croit un instant que la tour s'effondre, puis que les maudits chrétiens donnent l'assaut aux murailles [...] (Histoire du siège de Lisbonne (ci-après HSL), p. 172-3)

En (50a) comme en (50b), l'élément temporel antéposé (beau matin de juillet/manhã de Julho) n'a pas vraiment de fonction de repère. En effet, il «joue un rôle purement qualitatif qui consiste à [...] "donner corps", si l'on veut, à la situation fictive déjà décrochée implicitement de $\mathrm{Sit}_{0}$, par le simple fait qu'il s'agit d'un texte de fiction»(Chuquet, 1994: 53). Le décrochage fictif est donc posé d'emblée, et le renvoi aux événements situés dans ce plan décroché peut alors s'effectuer sans autre marquage de «passé» sur les formes verbales. Ces événements viennent s'inscrire, en fait, sur une espèce de toile de fond posée comme situation de référence par l'antéposition d'une localisation temporelle.

Mais en examinant la traduction française de plusieurs romans de José Saramago, auteur qui fait un usage abondant des transitions temporelles, dont certaines sont d'ailleurs déroutantes, nous pouvons constater que l'établissement du décrochage fictif peut prendre d'autres formes. La comparaison de l'énoncé (51a) qui suit avec sa traduction française nous montre clairement qu'il est possible de déclencher le récit d'événements au présent en posant le champ de la fiction par un procès aoristique au 'pretérito perfeito' (levantou-se) /au passé composé (s'est levé) qui permet, à lui seul, de créer la rupture nécessaire à l'introduction d'énoncés au présent dans le récit:

(51a) Raimundo Silva levantou-se da secretária, passeia no pequeno espaço livre do escritório, vem ao corredor para desafogar-se [...] da tensão de nova espécie que o está tomando, e em voz alta pensa, [...]. Cansou-se de percorrer o corredor, voltou ao escritório, mas não se sentou [...] (HCL, 1183)

(51b) Raimundo Silva s'est levé de sa table, il déambule dans l'espace réduit de son bureau, il va dans le couloir pour se décharger [...] de la tension d'une nouvelle espèce qui s'empare de lui et il réfléchit à haute voix. [...]. Il se lassa de parcourir le couloir, il retourna à son bureau mais ne s'assit pas [...] (HSL, 128-9) 
Autrement dit, cette rupture devient possible en insérant, tout simplement, des procès au présent dans une portion de récit au passé construite par le biais d'un enchaînement linéaire de procès/d'intervalles bornésfermés disjoints (Culioli, 1980: 187) dans lequel les événements révolus semblent racontés sur le mode du reportage en direct: les événements se déroulent sous les yeux d'un observateur fictif (façon de «faire entrer» le lecteur dans le vif du sujet) et, dans le texte portugais, c'est le présent progressif d'identification à un point de vue interne à la situation ([...] da tensão de nova espécie que o está tomando) qui est choisi pour évoquer une sorte d'arrêt sur la situation du personnage. Aspectuellement liée dans le récit au présent, la périphrase estar + 'gerúndio' s'oppose aux formes aoristiques du contexte environnant et vient, en fait, construire, par rapport à l'aspect globalisant aoristique des formes au présent simple (cf. supra, passeia, vem, pensa), une vision interne au procès repéré par identification à un point de vue subjectif. Cette périphrase "remplace" donc vraisemblablement le présent simple pour rétablir la distinction entre ouvert et fermé gommée par le système aoristique.

Bien que le français dispose d'une périphrase aspectuelle être en train de, le traducteur français n'a nullement cherché à traduire l'effet de mise en scène produit par le renvoi d'emblée, avec estar + 'gerúndio', à une situation actualisée. Et c'est d'ailleurs généralement le cas dans l'ensemble du roman, comme en témoigne un autre extrait du livre História do Cerco de Lisboa de José Saramago:

(52a) O cão aproximou-se outra vez, agora Raimundo Silva olha-o apreensivo, sabe-se lá se não estará raivoso, [...]. Raimundo Silva vai consultando os papéis, seguindo mentalmente o itinerário, e olha o cão a furto, e é então que se lembra da descrição que o historiador fez dos horrores da fominha [...], não ficou vivo nem cão, nem gato, até as ratazanas levaram sumiço, [...] (HCL, 1126)

(52b) Le chien s'approcha de nouveau, Raimundo Silva le regarde maintenant avec appréhension, il a peut-être la rage, [...]. Raimundo Silva consulte ses documents, suivant mentalement l'itinéraire, il regarde le chien à la dérobée et se souvient alors de la description que l'historien a faite des horreurs de la famine [...], pas un chien, pas un chat n'était resté en vie, même les rats avaient disparu, [...] (HSL, 71)

Tout comme dans les exemples (51a)-(51b) commentés plus haut, on assiste à un passage du passé au présent qui marque la rupture entre récit rétrospectif et récit-reportage présenté comme fictivement contemporain d'un observateur présent dans le récit. Il est à noter, une fois encore, que le passage du 'pretérito perfeito' au présent dans l'extrait de récit en portugais illustré par (52a) est reproduit spontanément dans la traduction proposée en $(52 \mathrm{~b})$, ce qui montre bien que l'hétérogénéité temporelle se rencontre dans les deux langues ${ }^{16}$. En revanche, la traduction française ne rend pas compte de l'alternance entre l'événementiel et le descriptif qui est explicitement marquée, en (52b), par la cooccurrence de deux formes aspectuelles: présent simple et périphrase ir + 'gerúndio'. Il semblerait, en effet, qu'en portugais, lorsque le présent simple est utilisé dans le récit, on rencontre fréquemment en alternance avec ces formes verbales au présent, des périphrases verbales à valeur progressive (par exemple, estar + 'gerúndio', ir + 'gerúndio') pour marquer les procès envisagés comme ouverts. En (52a), la forme vai consultando allie un procès télique au progressif véhiculé par ir + 'gerúndio'. Le progressif annule le telos inhérent à consultar os papéis, en introduisant une indétermination sur la fermeture de la situation. En effet, le progressif focalise sur le processus lui-même et ne dit rien sur la clôture du procès, bien que la valeur lexicale du verbe implique une culmination qui peut ou non se produire.

L'inexistence de ces périphrases en français est, on le verra, à l'origine de problèmes de choix de traduction, mais pour combler cette lacune, le traducteur aurait pu, tout de même, faire appel, en (52b), à la périphrase être en train de pour forcer une espèce d'arrêt sur image dans le récit, comme l'illustre le passage suivant que nous proposons:

(52b’) [...] Raimundo Silva est en train de consulter ses documents, [...] il regarde le chien [...] et se souvient alors de [...].

Une telle traduction serait plus spontanément interprétée comme correspondant au souci du traducteur d'homogénéiser les variations aspectuelles dans les deux langues. En effet, il nous semble que ce qui est 
alors particulièrement mis en relief, de façon encore plus nette avec être en train de + infinitif qu'avec ir + 'gerúndio', c'est l'instabilité de la situation-repère (consulter les documents), qui n'est posée que pour être aussitôt interrompue (donc déstabilisée) par l'irruption d'un événement (regarder le chien) qui déclenche par ricochet un autre événement: le personnage se souvient alors d'une histoire où même les chiens n'ont pas été épargnés par la famine. Le connecteur alors permet d'ailleurs d'opérer l'insertion de être en train de dans un repérage inter-énoncés: Raimundo est en train de consulter ses documents, mais en regardant le chien, il se souvient alors d'une histoire. Il s'agit bien d'un phénomène propre à être en train de qui, selon Franckel (1989: 67), a pour propriété de marquer un décalage entre la construction de $\mathrm{P}$ à partir d'un repère de type $\mathrm{S}$ et la localisation de ce même procès dans le temps. Pour représenter la dissociation entre ces deux formes de structuration (temporelle et notionnelle) qui renvoie au concept de «frayage» introduit pas Culioli, Franckel (1989: 65) propose le schéma suivant:

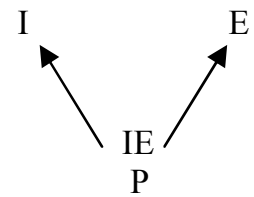

Figure 1. Concept de «frayage»

Selon Franckel (1989: 70), «l'interprétation de être en train de repose sur un champ de forces ${ }^{17}$ qui se constitue entre IE, position dans laquelle P fait l'objet d'un ancrage dans le temps, et I construit hors du plan temporel». Et c'est justement parce que cette périphrase «maintient dissocié le but et le cheminement: tant que I n'est pas atteint, E n'est pas écarté et les ponts avec IE ne sont pas coupés» (Franckel, 1989: 71) que être en train de peut fonder la valeur classique de «procès en cours» ${ }^{18}$.

Ainsi, si on décide de recourir à la périphrase être en train de + infinitif pour rendre compte de la valeur aspectuelle progressive qui sous-tend la forme verbale vai consultando dans l'énoncé Raimundo Silva vai consultando os papéis (cf. supra, ex. (52a)) $)^{19}$, on ne fait que signaler explicitement le hiatus entre la position IE et la position I. En effet, dans l'énoncé (52b') Raimundo Silva est en train de consulter ses documents que nous avons nous-mêmes constitué, l'atteinte de I (avoir fini de consulter les documents) devient, on l'a vu, fort incertaine car une pensée survient dans le temps et s'impose au personnage, de l'extérieur, comme à son insu. On peut dire que la localisation de $\mathrm{P}$ tend vers une conformisation à I dont l'atteinte est tout de même fortement compromise par l'irruption d'un P qui vient interrompre, du moins pour un moment, le «cheminement» du procès décrit en $(52 \mathrm{~b} ’))$.

Il est à noter que la valeur classique de «procès en cours» est parfois marquée, en français, par le biais de la périphrase être en train de + infinitif, bien que le présent simple vienne par défaut s'inscrire dans le mode de repérage déictique, avec les valeurs qui en découlent, à savoir celle qui marque une identification à $\mathrm{T}_{0}$, comme l'illustrent, par exemple, les énoncés suivants qui ont été extraits du roman $L a$ vie mode d'emploi (ci-après VME) de Georges Perec:

(53a) La femme regarde un plan qu'elle tient dans la main gauche (VME, 22)

(54a) Le troisième lit une lettre (VME, 48)

(55a) Il joue avec une petite toupie ronfleuse sur laquelle des oiseaux ont été dessinés (VME, 68)

(56a) Mme Albin commence à perdre la mémoire [...] (VME, 263)

Dans tous ces exemples, le présent donne l'illusion de se rapprocher de la forme être en train de + infinitif puisqu'il décrit bel et bien un événement en cours en $\mathrm{T}_{0}$. En effet, l'énoncé (53a) est proche de la femme est en train de regarder un plan [...], l'énoncé (54a) de le troisième est en train de lire une lettre et l'énoncé (55a) de il est en train de jouer avec une petite toupie ronfleuse. Comme le souligne, à juste titre, Franckel (1989: 76), il faut, tout de même, remarquer que ce que marque être en train de $e^{20}+$ infinitif est nettement plus complexe et spécifique que la valeur de procès en cours. Comme on l'a précisé plus haut, être en train de repose, en effet, sur un champ de forces qui se constitue entre IE et débouche donc 
facilement sur des valeurs analysables en termes de mise en garde (cf. supra, ex. (57)) ou d'intrusion imtempestive (cf. supra, ex. (58)) lorsque la situation décrite se construit relativement à une autre situation par rapport à laquelle elle se démarque:

(57) Attention à votre portefeuille! Il y a un papier qui est en train de tomber

(58) - Tu viens te promener avec nous?

- Laisse-moi tranquille, tu vois bien que je suis en train de travailler!

Il importe de rappeler, par ailleurs, que la périphrase être en train de est d'un emploi nettement plus contraint que estar $a+$ infinitif. Comme le précise Machado (1998) qui s'attache précisément à rendre compte des stratégies aspecto-temporelles adoptées, dans la VME, pour traduire, en portugais, des énoncés au présent du type de (53a)-(56a), la traduction de (59a) proposée par Pedro Támen montre, par exemple, que la contrainte de être en train de sur la périphrase à valeur inchoative commencer à + infinitif observée en (59a) ne se pose pas en portugais:

(59a) ?? Mme Albin est en train de commencer à perdre la mémoire [...]

(59b) A senhora Albin está a começar a perder a memória [...] (A vida modo de usar (ci-après VMU), p. 202)

Pour rendre compte de la dimension processive sous-jacente aux formes verbales contenues, par exemple, dans les énoncés $(53 a),(54 a)$ et $(55 a)$, la traduction portugaise fait nécessairement appel à la périphrase estar $a+$ infinitif:

(53b) A mulher está a olhar para uma planta que segura na mão esquerda (VMU, 21)

(54b) O terceiro está a ler uma carta (VMU, 38)

(55b) Está a brincar com um pequeno pião que ronca, no qual foram desenhados passarinhos [...] (VMU, 54)

On peut évidemment se demander s'il est possible de maintenir cette valeur progressive en remplaçant, en (54b)-(55b), le présent périphrastique par un présent simple. Comme le font remarquer Mateus et al. (2003: 144-5), le présent simple indique prioritairement l'habitualité lorsqu'il est combiné avec des procès de type dense (i.e. des procès atéliques [+ dynamiques], [- ponctuels]), ce dont témoigne l'emploi du verbe brincar dans cet exemple:

(55c) Brinca com um pequeno pião que ronca

Par ailleurs, le présent simple est rarement utilisé, en portugais, sans adverbiaux temporels et/ou aspectuels lorsqu'il côtoie des procès téliques, dans la mesure où l'on obtient des énoncés du type (54c) dont la situation décrite est difficilement interprétée comme ayant une occurrence singulière:

(54c) ?? O terceiro lê uma carta ${ }^{21}$

C'est pourquoi, on utilise, plus volontiers, pour marquer un repérage par identification par rapport à $\mathrm{T}_{0}$, la périphrase estar $a+$ infinitif (cf. supra, exs (54b) et (55b)); à l'instar de être en train de, cette périphrase portugaise sert à décrire une situation en déroulement et «ouverte», i.e. sans qu'il y ait inférence d'un aboutissement (Campos, 1998: 210); en revanche, l'emploi du présent simple français parvient, dans ce cas, à dénoter une situation singulière en cours en $\mathrm{T}_{0}$, comme l'illustrent les exemples (54a)-(55a) transcrits ci-dessus.

Il est important de souligner que les énoncés décrivant ce type de situation en français sont pour la plupart traduits par le biais de la périphrase ir $a+$ infinitif (tr. lit. 'aller + préposition $a+$ infinitif') lorsqu'ils font apparaître des verbes qui renvoient à un déplacement (de type de sortir, monter, descendre ${ }^{22}$. En effet, comme le fait remarquer Machado (1998), la comparaison des énoncés (59a)-(61a) qui suivent avec leur traduction portugaise (cf. infra, exs (59b)-(61b)) montre que celle-ci fait volontairement appel à une périphrase aspectuelle construite, elle-même, avec un verbe de mouvement (à savoir le verbe ir) pour 
exprimer clairement l'écart existant entre la localisation effective (là où l'on est) et l'objectif visé (là où l'on tend à aller) par les différents personnages:

(59a) Une femme [...] est en train de monter l'escalier (VME, 22)

(59b) Vai uma mulher [...] a subir a escada (VMU, 19)

(60a) Trois ouvriers sont en train de sortir de la pièce (VME, 48)

(60b) Vão três operários a sair da sala (VMU, 38)

(61a) Elle est en train de descendre l'escalier qui même à la loggia (VME, 65)

(61b) Vai a descer a escada que leva à loggia (VMU, 51)

Comme on l'a vu, c'est au prix de cet écart que être en train de peut fonctionner en français. Il n'y aurait aucune difficulté à utiliser, pour traduire (59a), (60a) et (61a), la périphrase portugaise équivalente, comme l'illustrent les trois exemples qui suivent:

(59c) Está uma mulher [...] a subir a escada

(60c) Estão três operários a sair da sala

(61c) Está a descer a escada que leva à loggia

mais il nous faut reconnaître que les périphrases construites avec ir (suivi soit d'un verbe à l'infinitif précédé de la préposition $a$, soit d'un verbe au 'gerúndio') semblent assez bien représentées dans le récit portugais au présent, tout au moins dans le corpus que nous avons étudié. Elles apparaissent surtout lorsqu'il s'agit de traduire des énoncés au présent contenant des indices contextuels (cf. infra, par exemple, petit à petit en (62a)) qui servent à décrire une situation où le procès semble être observé au ralenti cinématographique ${ }^{23}$.

A titre d'illustration, comparons la traduction de deux passages extraits de L'homme qui dort de Georges Perec qui ont pour temps de base le présent simple:

(62a) Tu bats les cartes, tu les étales, tu retires les quatre as, tu regardes le jeu. [...] Petit à petit le jeu s'organise, des contraintes apparaissent, des possibilités se font jour [...] (72-3)

(62b) Baralhas as cartas, expõe-las, retiras os quatro ases, observas o jogo. [...] Pouco a pouco, o jogo vai-se organizando ('va-se organisant'), vão aparecendo ('vont apparaissant') imposições, vão surgindo ('vont surgissant') possibilidades [...] (53)

(63a) Tu longes des champs de blé mûr, tu décapites des herbes folles à grands coups maladroits de ton bâton $[\ldots](40)$

(63b) Passas pelos campos de trigo maduro e desastradamente, com grandes bastonadas, vais decepando ervas daninhas (31)

Plutôt que de chercher à maintenir l'homogénéité temporelle des deux extraits qui précèdent, la traduction portugaise signale le besoin de dissocier plus nettement ce qui relève de l'événementiel (baralhas, expõe, retiras, observas dans l'exemple (62b)), passas dans l'ex. (63a)) et ce qui est l'ordre du descriptif (cf. supra, formes verbales en caractères gras), au moyen d'une alternance non pas temporelle mais aspectuelle. Les procès marqués par la périphrase aspectuelle $i r+$ 'gerúndio' occupent l'arrière-plan précisément parce que leurs bornes ne sont pas perçues, alors que l'aspect aoristique (exprimé ici par le présent simple) montre les bornes des procès, et les situe donc au premier plan.

\section{Considérations finales}

Dans les pages qui précèdent, nous avons essayé de présenter quelques résultats préliminaires d'une étude contrastive portant sur le présent simple français et ses équivalents portugais. La seule lecture de textes 
littéraires permet de voir que le présent peut, de par sa plasticité sémantique, s'adapter, à des degrés différents dans les deux langues, aussi bien aux valeurs temporelles d'antériorité que de postériorité, de simultanéité que d'itération, aussi bien aux valeurs aspectuelles non-bornées de l'imperfectif qu'aux valeurs bornées du perfectif.

Notre étude fait apparaître de frappantes ressemblances dans l'organisation du récit par rupture en français et en portugais: ce qui caractérise avant tout le présent, c'est la possibilité d'être repéré par identification par rapport à un repère, quel qu'il soit: il suffit donc d'avoir un repère spatio-temporel qui installe le révolu pour que le présent fonctionne dans le récit et, comme nous avons pu le montrer, les alternances passé-présent se retrouvent dans les deux langues. Ces variations temporelles constituent une forme de structuration du récit, permettant de mettre en valeur la saillance de tel ou tel événement, de produire un effet d'ouverture, puis de clôture d'une séquence événementielle.

Mais le présent portugais n'a pas les mêmes caractéristiques aspectuelles que le présent français dans la mesure où ce dernier peut, même lorsqu'il côtoie des procès denses discretisés (cf. ex. (54a)) ou non (cf. ex. (55a)) être interprété comme exprimant une valeur progressive. Un tel couplage, en portugais, ne semble autoriser qu'une lecture habituelle (cf. supra, exs (54c) et (55c)); pour que la valeur actuelle soit possible avec ce type de procès, le portugais se doit de faire appel au présent périphrastique (cf. exs (54b) et (55b)).

Il est bien clair, dans cette étude, que le présent simple s'emploie beaucoup moins en portugais qu'en français pour marquer une identification entre le moment du procès $\left(\mathrm{T}_{2}\right)$ et le moment de l'énonciation $\left(\mathrm{T}_{0}\right)$. Nous avons vu, en effet, que cette valeur d'identification n'est souvent possible que si le présent entre en cooccurrence avec des adverbiaux temporo-aspectuels et des périphrases verbales. Par ailleurs, le présent simple du portugais ne peut, à lui seul, marquer une valeur rétrospective. Pour construire cette valeur, il faut recourir, dans cette langue, à la périphrase acabar de + infinitif. En ce qui concerne la construction d'une valeur prospective, celle-ci dépend, on l'a vu, de la présence de déterminations contextuelles (interjections, adverbiaux temporo-aspectuels, ...) qui confèrent au présent simple une valeur futurale. Les contraintes qui pèsent sur le présent portugais sont directement fondées sur le fait que ce temps marque la mention du domaine notionnel associé à un prédicat, indépendamment de tout ancrage déterminé à la classe des $\mathrm{t}$. C'est l'incapacité à structurer la classe des t qui rend ce temps si fortement dépendant d'une série de structurations introduites de l'extérieur. Malgré les divergences rencontrées entre les deux langues en ce qui concerne les contextes d'apparition du présent simple, c'est à cette même caractérisation d'indifférenciation première de la classe des occurrences de t que fait appel Franckel (1989: 83) pour rendre compte du mode de fonctionnement du présent simple du français. Pour ce linguiste, ce temps est comparable, du point de vue des déterminations temporelles et aspectuelles, à l'infinitif. Comme le fait remarquer, à juste titre, de Vogüé (1993: 87), «le présent serait le bouche-trou du système verbal du français [...] il n'est pas marqué, parce qu'il ne marque rien». Et c'est justement ce qui le rend si malléable et donc si apte à marquer toutes les valeurs présentées ci-dessus.

En examinant des traductions portugaises de romans français, nous avons constaté, par ailleurs, que le présent simple français dans un récit est fréquemment traduit par autre chose que le présent simple, notamment par le biais de périphrases telles que estar $a+$ infinitif, ir $a+$ infinitif ou ir + 'gerúndio', qui ont pour effet de «densifier» le procès, en effectuant un repérage par rapport à un point de vue interne au procès, ce qui donne à ce dernier la valeur de processus en cours. Ces formes aspectuelles de présent progressif produisent ainsi un effet plus marqué d'arrêt sur image par contraste avec le présent simple français qui semble brouiller, dans son emploi monolithique dans un récit, les distinctions entre narration et description.

Les résultats de ce type d'étude contrastive basée sur des données attestées peuvent être utiles aux recherches linguistiques sur le temps aussi bien qu'aux ouvrages ayant des visées plus pratiques (notamment, dans le domaine de l'enseignement ou de la traduction). 


\section{Références bibliographiques}

Almeida, M. E. (2000). La deixis en portugais et en français. Louvain/Paris: Éditions Peeters.

Arrivé, M. et al. (1986). La grammaire d'aujourd'hui. Guide alphabétique de linguistique française. Paris: Flammarion.

Bach, E. (1981). On time, tense and aspect: an essay in English metaphysics. In Cole, P. (éd.). Radical Pragmatics. New York: Academic Press, 63-82.

Barroso, H. (1994). O aspecto verbal perifrástico em português contemporâneo. Porto: Porto Editora.

Benveniste, E. (1974). Problèmes de linguistique générale, vol. 2. Paris: Gallimard.

Borillo, A. (1991). De la nature compositionnelle de l'aspect. In Fuchs, C. (éd.), Les typologies de procès. Paris: Klincksieck, 97-102.

Bouscaren, J. \& Deschamps, A. (1991). Réexamen de la typologie des verbes anglais. Cahiers Charles V, 13. Travaux de linguistique énonciative, Université Paris 7, 7-23.

Bouscaren, J. \& Chuquet, J. (1987). Grammaire et textes anglais. Guide pour l'analyse linguistique. Paris: Éditions Ophrys.

Campos, M. H. C. \& Xavier, M. F. (1991). Sintaxe e Semântica do português. Lisboa: Universidade Aberta.

Campos, M. H. C. (1997). Para uma reinterpretação de alguns fenómenos aspectuais. In Campos, M.H.C. Tempo, Aspecto e Modalidade. Estudos de Linguística Portuguesa. Porto: Porto Editora, 183-196.

Campos, M. H. C. (1998). Dever e Poder um Subsistema Modal do Português. Lisboa: Fundação Calouste Gulbenkian/JNICT.

Chuquet, H. (1994). Le présent de narration en anglais et en français. Paris/Gap: Ophrys.

Comrie, B. (1976). Aspect. Cambridge: Cambridge University Press.

Culioli, A. (1980). Valeurs aspectuelles et opérations énonciatives: l'aoristique. In David, J. \& Martin, R. (éds), $L a$ notion d'aspect. Paris: Klincksieck, 181-193.

Culioli, A. (1990). Pour une linguistique de l'énonciation: opérations et représentations, tome 1, Collection HDL. Paris: Ophrys.

Culioli, A. (1991-92). Structuration d'une notion et typologie lexicale. A propos de la distinction dense, discret, compact. $B U L A G, 17,7-12$.

Cunha, C. \& Cintra, L. (1989). Nova Gramática do Português Contemporâneo. Lisboa: Edições João Sá da Costa.

De Vogüé, S. (1989). Discret, dense, compact: les enjeux énonciatifs d'une typologie lexicale. In Franckel, J.-J. (éd.), La notion de prédicat, collection ERA 642 (URA 1028). Paris: Université de Paris 7, 1-37.

De Vogüé, S. (1993). Des temps et des modes. Le Gré des Langues, 6, 65-91.

Desclés, J.-P. \& Guentchéva, Z. (1978). Construction formelle de la catégorie grammaticale de l'aspect. In David, J. \& Martin, R. (éds), La notion d'aspect. Paris: Klincksieck, 198-237.

Franckel, J.-J. \& Paillard, D. \& De Vogüé, S. (1988). Extension de la distinction discret, dense, compact au domaine verbal. In David, J. \& Kleiber, G. (éds), Termes Massifs et Termes Comptables. Paris: Klincksieck, 239-247.

Franckel, J.-J. (1989). Étude de quelques marqueurs aspectuels en français. Genève-Paris: Librairie Droz.

Franckel, J.-J. \& Paillard, D. (1989). Objet, complément, repère. Langages, 94, 115-127.

Franckel, J.-J. \& Paillard, D. (1991). Discret-dense-compact: vers une typologie opératoire. In Fuchs, C. (éd.), Les typologies de procès. Paris: Klincksieck, 103-136.

Fuchs, C. \& Gosselin, L. \& Victorri, B. (1991). Polysémie, glissements de sens et calcul des types de procès. In Fuchs, C. (éd.), Les typologies de procès. Paris: Klincksieck, 137-169. 
Gosselin, L. (1996). Sémantique de la temporalité en français. Un modèle calculatoire et cognitif du temps et de l'aspect. Louvain-la-Neuve: Duculot.

Guillemin-Flescher, J. (1981). Syntaxe comparée du français et de l'anglais. Problèmes de traduction. Paris: Ophrys.

Laca, B. (2005). Périphrases aspectuelles et temps grammatical dans les langues romanes. In Bat-Zeev, S. H. \& Le Querler, N. (éds), Les périphrases verbales. Amsterdam: John Benjamins, 47-66.

Lachaux, F. (2005). La périphrase être en train de, perspective interlinguale (anglais-français): une modalisation de l'aspect? In Bat-Zeev, S. H. \& Le Querler, N. (éds), Les périphrases verbales. Amsterdam. John Benjamins, 119142.

Machado, E. M. do N. (1998). La vie mode d'emploi - a vida modo de usar; a tradução modo de abusar?. Dissertation de 'Mestrado' non publiée, Universidade do Minho.

Mateus, H. M. M. et al. (1983). Gramática da língua portuguesa. Coimbra: Editorial Caminho.

Moens, M. \& Steedman, M. (1988). Temporal Ontology and Temporal Reference. Computational Linguistics, 14, 2 , $15-28$.

Paillard, D. (1988). Temps, aspect, types de procès: à propos du présent simple. Recherches nouvelles sur le langage, Université de Paris 7, Coll. ERA 642, 92-107.

Pusch, C. D. (2003). La grammaticalisation de l'aspectualité: Les périphrases à valeur progressive en français. Verbum, 4, 495-508.

Serbat, G. (1988). Le prétendu 'présent' de l'indicatif: une forme non déictique du verbe. L'information grammaticale, 38, 32-35.

Vendler, Z. (1967). Linguistics and Philosophy. Ithaca, N.Y.: Cornell University Press.

Wagner, R. L. \& Pinchon, J. (1962). Grammaire du français classique et moderne. Paris: Hachette Université.

\section{Corpus littéraire français/portugais}

Saramago, J. (1991). História do cerco de Lisboa. In Obras de José Saramago, volume III, Porto, Lello \& Irmão Editores, 1063-1394.

Leibrich, G. (1992). Traduction française, Histoire du siège de Lisbonne. Paris: Editions du Seuil.

Perec, G. (1978). La vie mode d'emploi. Paris: Hachette.

Tamen, P. (1989). Traduction portugaise, A vida modo de usar. Lisboa: Editorial Presença.

Perec, G. (1990). L'homme qui dort. Gallimard, Collection Folio.

Figueiredo, M. J. V. (1991). Traduction portugaise, Um homem que dorme. Lisboa: Editorial Presença.

\footnotetext{
${ }^{1}$ Comme méthode de travail, nous adopterons celle proposée par Guillemin-Flescher (1981) dans son examen différentiel du français et de l'anglais. La méthode/démarche consiste à travailler sur un corpus traduit, et plus concrètement sur un échantillon d'œuvres littéraires de langue française et portugaise traduites d'une langue à l'autre afin de pouvoir examiner les opérations mises en œuvre dans chacune des deux langues, avec une base de départ commune. Et cela permet également de voir par quelles restructurations et par quels marqueurs le traducteur parvient à faire passer, dans la langue cible, les opérations qui sont à l'origine du texte source.

${ }^{2}$ Sans donner le détail des caractéristiques sur lesquelles fonctionne cette typologie tripartite, nous préciserons simplement que les procès /compact/, /discret/, /dense/ se laissent ainsi définir: i) les discrets qui, à la différence des denses et des compacts, sont notionnellement téliques, correspondent à des procès à bornes confondues (du type sortir, tomber), qui se caractérisent par la construction d'un procès qui "a valeur de transition non décomposable entre un état initial et un état résultant" (Bouscaren et al,. 1993: 14). Cela signifie que le début du procès implique en même temps le terme du procès. Mais les discrets peuvent aussi renvoyer à des procès à bornes séparables (ou disjointes) comme construire, réparer, dont la télicité semble plus ou moins inhérente aux propriétés primitives du procès en jeu; ii) les denses correspondent à des procès à bornes séparables atéliques qui peuvent néanmoins faire l'objet, à un niveau ultérieur, d'une délimitation "construite": par exemple, au moyen d'un formatage extrinsèque lié à la présence d'un objet (ex. il a bu son café) (cf. Franckel et Paillard, 1989). Comme le remarque, très justement, de Vogüé (1989: 1), "la détermination de l'objet suffit à doter le procès d'une borne d'accomplissement (externe), et donc d'un format (extrinsèque)"; iii) les compacts qui ne sont, à proprement parler, pas discrétisables correspondent aux procès non bornés (ou procès
} 
dits statifs): le propre de ces procès tient donc au fait que la propriété être $P$ n'a aucune «forme» indépendamment de celle que peut conférer sa temporalisation. Mais comme le précisent Bouscaren \& Deschamps (1991: 12), il faut bien comprendre qu'un verbe n'appartient pas «une fois pour toutes à tel ou tel type». Dans son étude portant sur la nature compositionnelle de l'aspect, Borillo (1991) précise, en effet, que «la catégorisation ne peut s'appliquer, [...] uniquement au vu de la classe lexicale du verbe, mais doit attendre l'étape de la mise en fonctionnement du verbe dans une phrase», car tous les opérateurs utilisés (temps, complémentation, détermination, ...) vont contribuer à la caractérisation aspectuelle de l'énoncé.

${ }^{3}$ En effet, comme le notent, à ce propos, Bouscaren et Deschamps (1991: 9), les verbes étiquetés «activity» ou «accomplishment» le sont souvent en fonction de la complémentation ou des variables aspectuo-temporelles. Ainsi, le classement de Vendler repose sur des exemples comme run, paint (activity) en face de run a mile, paint two doors (accomplishment). Ces exemples montrent qu'il y a manisfestement confusion entre la valeur notionnelle qui relève de l'item lexical choisi et ce qui découle de l'utilisation de cet item dans un énoncé.

${ }^{4}$ Bien sûr, il va y avoir interaction entre les types de procès et le présent simple. Si on le combine avec des procès de type dense (i.e. à bornes séparables atéliques) le présent tend à renvoyer à une interprétation actualisée (cf. supra, exs (4) Ne le dérange pas, il téléphone (il est au téléphone); (6) Chut! Pierre dort (= «Pierre est en train de dormir»); alors que si l'on croise le présent simple avec un procès à bornes confondues comme partir, on a alors affaire à un contexte prospectif, même sans qu'aucun adverbe temporel soit explicite, comme en témoigne l'exemple (11c) proposé ci-dessus: (11c) je pars qui, à l'instar de (11b) je pars demain matin, tend à s'interpréter comme: j'ai l'intention de partir ou bien je menace de partir. Dans ces deux exemples ((11b)-(11c)), le procès est construit comme validable (cf. Culioli, 1980: 185) et non comme validé en $\mathrm{T}_{0}$. Mais le présent simple peut être tout aussi compatible, comme on peut le constater, avec une valeur itérative (cf. supra, ex. (11a)), ou bien encore une valeur aoristique (cf. supra, ex. (13)).

${ }^{5}$ Il est intéressant de noter que le croisement du présent avec deux verbes qui réfèrent a priori à des activités d'ingestion dont l'une concerne les éléments liquides (boire), l'autre les éléments solides (manger) débouche sur des valeurs différentes (Franckel, 1989: 48). Alors que dans (3a) Pierre boit, boire relève d'un fonctionnement compact dans la mesure où il n'y a pas, dans ce cas, construction d'occurrence, mais prédication d'une propriété (Pierre est le support à travers lequel s'appréhende la propriété être buveur) qui ne présente aucune détermination singularisante sur le plan des $t$, dans (2a) Pierre mange, manger relève d'un fonctionnement dense, en ce sens où (2a) marque simplement que $\mathrm{P}$ est localisable sur la classe des t. Dans Pierre mange, ce qu'il mange n'est défini qu'au travers même de ce qu'il mange au fur et à mesure de son repas.

${ }^{6}$ La plupart des exemples qui illustrent les valeurs du présent simple en français sont empruntés à Paillard (1988: 102).

${ }^{7}$ Les exemples (14), (18), (19), (21) et (23) ont été retirés de la grammaire de Celso et Cunha (1989: 448).

${ }^{8}$ Le concept d'aoristique a été introduit par Culioli (1980: 189) dans sa théorie du repérage pour rendre compte de toute une série d'énoncés ayant en commun une relation de rupture entre la situation validant le procès à repérer et toute autre situation, y compris la situation d'énonciation (rappelons que la valeur de rupture prise par l'opérateur de repérage est notée: $\omega$ ). Plusieurs temps peuvent prendre cette valeur en français, en particulier le présent dit «historique» ou de «narration», qui est, en effet, un exemple du mode de repérage aoristique. Voir également la rubrique consacrée à ce concept dans le «Glossaire analytique» de Bouscaren et Chuquet (1987: 165-166) ou bien encore Desclés et Guentchéva (1978: 221).

${ }^{9}$ Il faut préciser, en effet, qu'il est possible de traduire littéralement en portugais des exemples du type de (6) qui mettent en jeu des procès denses du type de travailler (ex: (6') atualmente, trabalha (tr. lit. 'actuellement, (il) travaille')), mais trabalha ne prend pas, dans ce cas, une valeur de «procès en cours en $\mathrm{T}_{0}$ » mais établit plutôt une relation de contraste par rapport à une période de temps antérieure (où le sujet de l'énoncé ne travaillait pas).

${ }^{10} \mathrm{Il}$ est bien connu que le portugais, à l'instar d'autres langues romanes comme l'espagnol (cf. Pusch, 2003; Laca, 2005), fait usage de constructions dites «périphrastiques» à valeur aspectuelle, où le 'gerúndio', de forme -ndo (cantando, dizendo, partindo...) en portugais, est régi par de nombreux auxiliaires (estar, andar, ir, vir ...) représentatifs d'une permanence, d'une durée, etc. Précisons, tout de même, que l'emploi du 'gerúndio' dans ces constructions périphrastiques est plus fréquent dans la norme portugaise du Brésil que dans celle du Portugal. Dans son ouvrage entièrement consacré à l'aspect périphrastique, Barroso (1994: 88-108) montre que les périphrases verbales disponibles en portugais peuvent correspondre à six «visions» différentes: parallèlement à une «vision angulaire» (représentée par estar $a+$ infinitif ou estar + gerúndio), ce linguiste considère qu'il existe également des visions «rétrospective», «prospective», «continuative», «extensive» et finalement une vision qu'il désigne de «comitiva».

${ }^{11}$ Il nous faut évoquer ici la différence de comportement entre être en train de + infinitif et estar a + infinitif car il semblerait que cette dernière périphrase côtoie bien plus naturellement les procès de type compact (i.e. qui sont statifs dans leur interprétation de base) en portugais qu'en français. Considérons, par exemple, la différence d'attestabilité entre: (a) Ele está a ser desagradável! et (a') ??Il est en train d'être désagréablel! (b) A Ana está a viver em Paris et (b') ?? Ana est en train de vivre à Paris; (c) O Pedro está a gostar da Maria et (c') *Pedro est en train d'aimer Marie. La possibilité d'occurrence de la périphrase progressive avec des verbes statifs (compacts) en portugais engendre, dans la terminologie de Bach (1981), la construction d'une situation stative temporaire. Évidemment, parmi ces prédicats, seuls ceux qui acceptent des opérations supplémentaires de délimitation peuvent être associés, en portugais, à la périphrase estar a + infinitif. Les exemples du type (d): A Maria está a ser grande; (d') Maria est en train d'être grande sont aussi peu naturels en portugais qu'en français. On notera, tout de même, que les procès /compact/ sont, à certaines conditions, susceptibles d'une «décompactisation» en français, notamment si on introduit une visée (une forme d'agentivité) (Attention, là tu es en train d'être franchement désagréable et plus encore Il est en train de se montrer désagréable). 
${ }^{12}$ Le portugais, tout comme le castillan, a deux formes verbales pour traduire le français être - estar et ser: (i) estou em Lisboa (je suis à Lisbonne), (ii) sou português (je suis portugais). Estar est l'auxiliaire du présent périphrastique en portugais.

${ }^{13}$ En effet, le marqueur já est très productif dans la construction du futur proche: espere um pouco ('attendez un peu'): já faço isso (tr. lit. 'déjà (je) fais ça'); já o atendo (tr. lit. 'déjà (je) vous réponds'); já lhe falo (tr. lit. 'déjà (je) vous parle'). Comme le note Campos (1997: 64), il est possible de remplacer já par l'adverbe imediatamente ('immédiatement') pour marquer cette valeur prospective: já limpo (tr. lit. 'déjà (je) nettoie'); limpo imediatamente (tr. lit. '(je) nettoie immédiatement').

${ }^{14}$ Le présent simple ne peut avoir, en portugais, une valeur rétrospective que s'il est associé au verbe vir (en fr. venir): Venho da praia. Dans un contexte du genre question-réponse, cet énoncé peut fort bien constituer la réponse à la question qui suit: «então de onde vens?» (en fr.: alors d'où viens-tu?)? Venho da praia (acabei de chegar da praia) (en fr.: je viens de la plage; je viens d'arriver de la plage).

${ }^{15}$ En effet, comme le souligne Gosselin (1996: 195), l'association du présent simple à un procès intrinsèquement ponctuel donne lieu à une multiplicité d'effets de sens, notamment à celui du «futur proche» (cf. supra, note 4) ou du «passé immédiat» (cf. supra, exs (37a) ou (38a)).

${ }^{16}$ Il est à noter que la traduction française du passage (51a) augmente l'hétérogénéité temporelle de ce dernier: en effet, on assiste, dans le texte d'arrivée, tout d'abord, à un passage du passé composé (s'est levé) au présent (déambule, va, s'empare, réfléchit), puis à une alternance du présent au passé simple (se lassa, retourna, s'assit) alors que dans le texte de départ, on n'est confronté qu'à un seul temps du passé, à savoir le 'pretérito perfeito simples' (levantou-se, cansou-se, voltou, se sentou) car, ce temps verbal «utilisé en portugais de la même façon que le perfectum en latin, cumule en quelque sorte les valeurs du parfait et de l'aoriste grecs. Cela explique que ce pretérito traduise aussi bien le passé simple (aspect aoristique ou synoptique) que le passé composé (aspect accompli ou rétrospectif)» (Almeida, 2000: 266). Il est tout de même curieux de voir qu'un extrait aussi court (cf. (51b)) parvient à combiner deux temps du passé qui renvoient à deux modes différents de construction du récit: un passé composé qui est à la base d'un récit rétrospectif construit à partir de $\mathrm{T}_{0}$, et un passé simple qui se situe intégralement dans le plan du récit en rupture par rapport à $\mathrm{T}_{0}$. Il semblerait, en tout cas, que les deux langues compensent la neutralité temporelle du présent, et en même temps en tirent parti, en multipliant les variations temporelles, et en favorisant un va-et-vient constant entre les deux pôles que représentent «le passage du récit construit par translation dans le révolu au récit construit par identification par rapport à un repère décroché» dans la succession événementielle (Chuquet, 1994: 203).

${ }^{17}$ Et justement parce que la forme être en train de + infinitif met clairement en évidence le principe d'une discordance entre $\mathrm{P}$ (entre le à faire) et I (l'effectivement fait) qu'elle fonctionne mieux avec des adverbes à valeur d'appréciation négative (cf. Franckel, 1989: 76). En effet, on admettra bien volontiers que il est en train de bien lire bien maintenant est bien moins naturel que il est en train de lire de travers ou que il est en train de bien danser est nettement moins bien toléré que il est en train de danser comme un sabot. On notera qu'avec le présent simple, l'emploi d'adverbes qui tendent à marquer une conformité ne pose aucun problème d'attestabilité: il lit bien; il danse bien sont parfaitement acceptables, ce qui prouve bien que le présent simple a un comportement différent de être en train de.

${ }^{18}$ De ce point de vue, on peut dire que le fonctionnement du passé composé est inverse de celui de être en train de avec lequel il est d'ailleurs strictement incompatible: alors que être en train de + infinitif implique une ouverture de la sous-classe de t localisatrice de $\mathrm{P}$, le passé composé marque par définition une fermeture et une hétérogénéité de la classe des $\mathrm{t}$, au sens où il y a nécessairement une discontinuité entre $\mathrm{T}_{0}$ et le (ou les) instant(s) de localisation du procès (qui est alors nécessairement situé par rapport à ce ou ces $\mathrm{t}$ distingué(s) $\left(\mathrm{T}_{2} \neq \mathrm{T}_{0}\right)$ ). Il a été en train de $P$ marque donc une contradiction entre la fermeture imposée par le passé composé et l'ouverture imposée par être en train de + infinitif. On notera, toutefois, qu'une telle contradiction n'existe pas dans toutes les langues romanes, notamment en portugais. En effet, comme le remarque à juste titre Campos (1997: 189) à qui nous empruntons d'ailleurs l'ex. suivant: esta manhã, o João escreveu/esteve a escrever relatórios, le portugais a à sa disposition deux formes alternatives: escreveu et esteve a escrever, alors que le français ne connaît pas une telle oppostion (il a écrit/*a été en train d'écrire des rapports) (cf. Laca, 2005: 51) pour les raisons évoquées ci-dessus.

${ }^{19}$ Alliée au marqueur de repérage anaphorique então, qui reprend, en (52a), les paramètres associés à «olha o cão», la périphrase ir + 'gerúndio' instaure, dans la relation inter-énoncés, une relation de cause à effet entre le regard posé par le personnage et la mise en place d'un retour en arrière par le biais de la mémoire du personnage, et plus concrètement par le biais d'un verbe exprimant le souvenir (se lembra/se souvient). Et c'est à partir de la valeur progressive de la forme verbale vai consultando que va se construire une sorte de tableau statique dans lequel on assiste à l'introduction du récit-souvenir, détaché du récit primaire, de la chronologie narrative. Il est bien évident que dans tous les cas où $i r$ + 'gerúndio'est rendu par le présent simple en français, on assiste «à une perte dans la distinction entre énoncés narratifs et descriptifs et un repérage moins marqué par rapport à un point de vue [...]» (Chuquet, 1994: 126) aspectuel marqué par ir + 'gerúndio'. En effet, le procès consulter est, dans l'exemple (52b), déterminé comme étant «pris en bloc», «vu de l'extérieur», inséré dans une série d'événements ponctuels (on ne s'intéresse pas au déroulement interne du procès en soi), d'où la traduction par le présent simple (Raimundo Silva consulte ses documents [...]), alors qu'en (52a), ce même procès est interprété comme un «ouvert». En effet, la forme ir + 'gerúndio' marque le repérage de ce procès par rapport à un point de vue repère posé par la proposition e olha o cão a furto, et correspond à une relation de concomitance et non de succession entre les procès (consultar et olhar), ce qui favorise l'interprétation descriptive.

${ }^{20}$ Comme le note, en effet, Lachaux (2005: 126), la périphrase être en train de marque «un filtrage énonciatif, met en alerte l'attention du co-énonciateur, et lui signale explicitement un lien avec la situation d'énonciation». En effet, en (57), être en train de marque non pas un processus de chute (du papier) mais une mise en garde contre un risque de chute (cf. Franckel, 1989: 74). 
Attention! signale clairement l'inadvertance. Dans un dialogue du type de (58) qui s'inscrit dans un contexte d'interruption intrusive, la proposition faite par le premier locuteur (il s'agit de I de Q: aller te promener) est reconstruite comme mauvaise valeur par rapport à $\mathrm{P}$ (travailler), i.e. comme menant à $\mathrm{E}(\mathrm{de} \mathrm{Q})$. Dans ces cas de figure, on réintroduit donc les conditions d'un «frayage» qui rendent possible l'emploi de être en train de. Comme le notent fort bien Franckel et Paillard (1991: 121), cette discordance peut, bien évidemment, se solder par un simple décalage temporel, i.e. par un décalage entre une visée et une réalisation dans le temps. C'est ce qui se produit dans les exemples du type procès en cours (ex. je suis en train de rédiger le rapport que tu m'as demandé).

${ }^{21}$ Pour récupérer l'acceptabilité de (54c), il nous faut, en effet, faire ressortir l'aspect itératif de la situation décrite: (54c') O terceiro lê uma carta todas as semanas (tr. lit. 'le troisième lit une lettre toutes les semaines').

${ }^{22}$ En effet, comme le précise, à juste titre, Machado (1998), l'emploi de la périphrase $i r a+$ infinitif aboutit à de véritables inacceptabilités lorsque l'on essaie de la combiner à des verbes dépourvus de cette valeur de dynamicité. Faire appel à cette périphrase pour traduire, par exemple, un énoncé du type: (a) Debout devant la loge, une femme est en train de lire la liste des habitants de l'immeuble (p. 113) serait fort peu naturel: (b) *De pé diante do cubículo, vai uma mulher a ler a lista dos habitantes do prédio. Aussi n'est-il pas étonnant de voir que l'emploi de être en train de doit être reproduit spontanément dans la traduction portugaise: (c) De pé diante do cubículo está uma mulher a ler a lista dos habitantes do prédio (p. 91). Par contre, si l'on introduit l'idée de déplacement dans la situation décrite en (b), en modifiant, par exemple, le repère de localisation spatiale qui est antéposé (De pé diante do cubículo $\rightarrow$ de pé no autocarro ('debout dans le bus'), ir a + infinitif devient tout à fait acceptable: De pé no autocarro, vai uma mulher a ler a lista dos habitantes do prédio.

${ }^{23}$ En effet, comme le notent Cunha \& Cintra (1989: 395), ir s'associe au 'gerúndio' pour indiquer la réalisation graduelle d'une action, en signalant qu'elle se réalise progressivement (o navio ia encostando (pouco a pouco) $\rightarrow$ tr. lit. le navire allait accostant (реи à peu)) ou par étapes successives (os convidados iam chegando de automóvel (sucessivamente) $\rightarrow$ tr. lit. les invités allaient arrivant en automobile (successivement)). 